\title{
Contrasting RNN-based and simulation-based models of human physical parameter inference
}

\author{
Anonymous CogSci submission
}

\begin{abstract}
A number of recent studies have used ideal observer models to capture human physical learning and reasoning as based on approximate mental simulation. While these approaches can match human competence in specific tasks, they are still relatively far from cognitive plausibility and are limited in their ability to capture patterns of human errors. In the current work, we train a recurrent neural network on the same physical reasoning task explored in Bramley, Gerstenberg, Tenenbaum, and Gureckis (2018) (passive condition), finding a closer match to human patterns than the ideal observer model previously used to make sense of the human judgement patterns.

Keywords: intuitive physics; mental simulation; recurrent neural networks
\end{abstract}

\section{Introduction}

Developmental studies suggest that children progressively learn to reason about their physical surroundings. For example, at as young as six months we have expectations about physical rigidity (Hespos, Ferry, \& Rips, 2009) and continuity-i.e. that objects do not disappear arbitrarily (Spelke, 1990). By 12 months, we can anticipate movement and the results of collisions (Baillargeon, $\mathrm{Li}, \mathrm{Ng}, \&$ Yuan, 2009). By adulthood, humans are uniquely competent physical reasoners, outstripping the cutting edge in $\mathrm{AI}$ in terms of capacity to plan in and navigate through complex and heterogeneous physical environments. For example, adults make accurate intuitive judgements about the stability of towers of objects (Battaglia, Hamrick, \& Tenenbaum, 2013) and can predict the trajectories of moving objects (Smith, Dechter, Tenenbaum, \& Vul, 2013). Unlike our nearest genetic neighbours (Van Schaik, Deaner, \& Merrill, 1999), we have a sophisticated ability to find or make tools to solve physical puzzles and challenges (Allen, Smith, \& Tenenbaum, 2019). Although see also Mccloskey, Caramazza, and Green (1980) for evidence of our limitations.

How exactly we achieve these feats is still a matter of debate. Some have argued that adult competency in physical reasoning implies that we learn a probabilistic generative model of everyday physics that we then use to simulate relevant situations (Hamrick, Battaglia, Griffiths, \& Tenenbaum, 2016). Such accounts of this ability have largely been cast at Marr's computational level (Marr. 1982) and, given the uncertainty inherent to everyday physical reasoning, many leverage sampling over many independent simulations to approximate probabilistic inference. One source

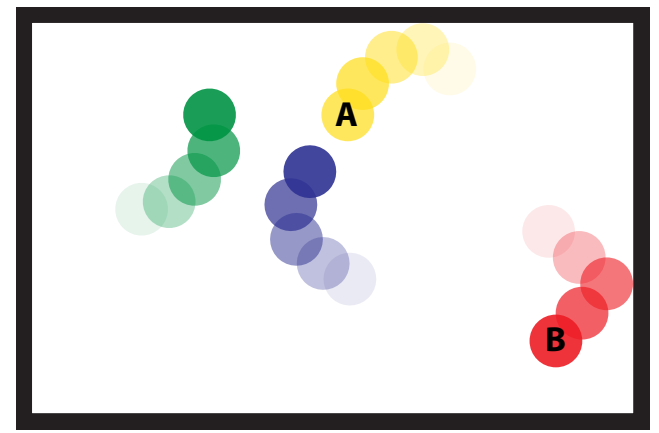

Figure 1: A visualization of the simulated world. Two target pucks labelled 'A' and 'B' and two distractor pucks. Colors are randomly assigned.

of uncertainty is perceptual imprecision of objects' locations and extents. But another is that accurate physical simulation depends on knowledge of various latent parameters that determine how a novel physical object will behave and interact with others-i.e. its mass, texture, elasticity and with respect to forces like gravity and magnetism. These properties can be inferred on the basis of evidence. On the ideal observer account, this is achieved by sampling parameter settings from a prior and upweighting settings that minimise the divergence between simulations and observations (Bramley et al. 2018; Ullman, Stuhlmüller, Goodman, \& Tenenbaum 2018). However, other authors have flagged the limitations of this approach as a characterisation of cognitive processing (Davis \& Marcus, 2015). One issue is that aggregating over many independent simulations is computationally inefficient, but it is not clear how else simulations, which are necessarily precise, can incorporate uncertainty. Relatedly, the aggregation over simulations approach seems unable to account for certain systematic errors in certain physical reasoning tasks. For example, Ludwin-Peery, Bramley, Davis, and Gureckis (2019) found that, for certain seemingly simulable physical inference problems, people were systematically fooled. For example, in an experiment with block towers, participants chose several physically impossible outcomes with a superficial resemblance to starting states over those that would result from an accurate mental simulation. In another study, people systematically reported that a specific compound outcome was more probable than one of its constituents, known as the conjunction fallacy (Ludwin-Peery, Bramley, Davis, \& 
Gureckis, in revision). Some authors have taken this mixed evidence to suggest we use a hybrid approach mixing simulation with model-free reasoning (Smith et al., 2013, Ullman et al. 2018). Here we consider whether human learning patterns can be well accounted for without an explicit simulation model.

Recent machine learning approaches have aimed at achieving human-level physical reasoning using both modelbased and model-free approaches. Battaglia, Pascanu, Lai, Rezende, and Kavukcuoglu (2016), for instance, equip probabilistic models capable of simulation with neural networks to reason about object properties, such as their potential energy, and trajectory. These tasks, however, are too tedious or detailed to be performed by humans and thus not ideal for providing insight into the human model of physical reasoning. On the other hand, studies such as Mottaghi, Bagherinezhad, Rastegari, and Farhadi (2015) have tested the abilities of a fully model-free neural network approach at predicting the trajectory of objects from images with successful results. Nevertheless, it would be difficult to evaluate and compare human reasoning abilities through these tasks as they do not map well onto everyday physical reasoning contexts.

Bramley et al. (2018) explored a physical reasoning task in which participants had to identify the relative masses and magnet-like forces of several "pucks" in an enclosed 2D environment akin to an air hockey table, that behaved in line with Newtonian physics (see Figure 1). In their passive condition, participants watched 45 second clips in which the pucks moved around the scene colliding and exerting magnet-like forces on one another. Participants in this condition found the task difficult and identified masses correctly $46 \pm 29 \%$ and forces correctly $61 \pm 22 \%$ of occasions (relative to a chance level of $33 \%$ and higher performance when they were allowed to intervene and move the objects themselves). Building on Ullman et al. (2018), the authors used an ideal observer model that, having access to the underlying physics simulator, would simulate forward all the possible mass and force configurations and compare their results to the observed trajectories using this to derive a posterior probability distribution over parameters. Using this model, they computed several metrics of the difficulty of each trial including entropy and a frame-by-frame evidence measure they called predictive divergence. While these measures were useful in characterising behaviours in the active conditions, they were not closely aligned with trial by trial difficulty. Furthermore, all the measures suggested the clips were more informative with respect to the mass questions than the force questions while participants were markedly more accurate at identifying the forces.

In this work, we approach this task using a model-free neural network approach. In particular, we train recurrent neural networks (see Salehinejad, Sankar, Barfett, Colak, \& Valaee, 2017, for a survey) to classify masses and forces based on observing clips. As we will show, this approach aligns much better with participants in Bramley et al.(2018) original study and a new sample of 100 participants facing 36 new passive learning problems in the same environment. Our RNN approach not only achieves a comparable overall accuracy to participants, but also better correlates with participants patterns of accuracy. Lastly, we explore the trained network's dynamics to infer which intervals in the simulation are most informative. We show that, while correlated with the ideal observers' informativeness metric developed by Bramley et al. (2018) for some examples, the RNN appears to misunderstand and confuses evidence in similar situations to humans. We end by commenting on the implications of these results for understanding human physical reasoning.

\section{Methods}

\section{The environment and the task}

The environment, simulated using Box2D, consists of four pucks that move inside of a $4 \times 6 \mathrm{~m}$ box that collide with, attract and repel each other following Newtonian physics. Two appear labelled with a letter (A or B) and are referred to as target pucks while the other two are unlabelled and serve as distractors. Each simulation is characterised by the mass of the target pucks, to choose from $[1 \mathrm{~kg}, 1 \mathrm{~kg}],[1 \mathrm{~kg}, 2 \mathrm{~kg}]$ or $[2 \mathrm{~kg}$, $1 \mathrm{~kg}$ ], and the magnetic force between every pair of pucks, one of $\left[3 m / s^{2},-3 m / s^{2}, 0 m / s^{2}\right]$. This gives a total of $3^{7}$ possible configurations. Given the environment, the participant is tasked with answering questions regarding the physical properties of the target pucks by watching a simulation of $45 \mathrm{~s}$. For the mass questions, the participants have to guess which target puck is heavier ('A is heavier', 'B is heavier' or 'same') while in the force questions they must discern whether there exists any magnetic force between them ('attract', 'repel' or 'none').

Training datasets and data processing: The $2187\left(3^{7}\right)$ possible world configurations were divided into three groups: $70 \%$ for training, $15 \%$ for validation and $15 \%$ for test. A trial is generated by combining one of these configurations with sampled starting conditions of the pucks (positions and velocities). This means that, even though the number of configurations is limited, the number of different trials is unbounded. Following this approach, 3500 trials were simulated for the training, 1000 for the validation and 1000 for the test dataset. By default, the simulator captures the position $(x, y)$ and the velocities $(v x, v y)$ of each puck at a rate of $60 \mathrm{fps}$, making a total of 2700 steps per simulation. The utility of other features such as the distance and angle (its cosine and sine) between pucks was also be tested and compared with the baseline. Additionally, different sequence lengths (45s, 30s and 15s) and resolutions (60fps, 30fps and 20fps) were compared.

Participant dataset: 100 participants were recruited using Amazon Mechanical Turk (36 female, age 35.6 \pm 10.5 ) and subdivided in four groups of 25 participants. Each group faced a different set 11 trials, including two practice trials, making a total of 36 test trials. For each trial, participants submitted a mass and a force answer as well as a value for the confidence. Participants were paid $\$ 1.50$ plus performance 


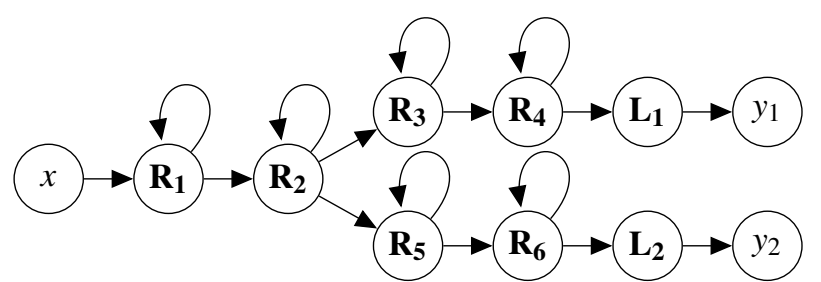

Figure 2: The Y-shaped RNN architecture used. $R_{i}$ and $L_{i}$ denote the recurrent and linear layers respectively. The input sequence $(x)$ and networks' outputs $\left(y_{1}\right.$ and $\left.y_{2}\right)$ also included.

bonuses $(\$ 2.46 \pm 0.74)$ and the task took $21 \pm 9.6$ minutes.

\section{Recurrent Neural Networks}

We use Recurrent Neural Networks (RNN) to tackle this task. RNNs hold a hidden state vector that is updated every timestep as a function of its previous value and the current input. This vector can then be used to produce an output that takes into account the whole sequence rather than just the latest timestep. Depending on how this hidden state is updated, we can differentiate between types of RNN layers, such as Gated Reccurent Units (GRU) (Cho, van Merrienboer, Bahdanau, \& Bengio, 2014). GRUs use gates to update the hidden state vector. The reset $\left(r_{t}\right)$, update $\left(u_{t}\right)$ and new $\left(n_{t}\right)$ gates are updated every timestep as shown below:

$$
\begin{aligned}
r_{t} & =\sigma\left(W_{i r} x_{t}+W_{h r} h_{t-1}\right) \\
u_{t} & =\sigma\left(W_{i u} x_{t}+W_{h u} h_{t-1}\right) \\
n_{t} & =\tanh \left(W_{i n} x_{t}+r_{t} *\left(W_{h n} h_{t-1}\right)\right)
\end{aligned}
$$

where $\sigma$ denotes the sigmoid function and tanh the hyperbolic tangent. The hidden state vector is then updated as a weighted average between the previous hidden state value, $h_{t-1}$, and the current input, through the new gate $\left(n_{t}\right)$ :

$$
h_{t}=\left(1-u_{t}\right) * n_{t}+u_{t} * h_{t-1}
$$

In multilayer or stacked RNN architectures, the sequence of hidden states $\left(h_{0}, h_{1}, \ldots, h_{t}\right)$ is fed into subsequent recurrent layers that can further process the input to obtain higherlevel features. The set of weights $\left(W_{i r}, W_{h r}, W_{i u}, W_{h u}, W_{i n}\right.$ and $W_{h n}$ ) is trained using Backpropagation Through Time (BPTT) (Werbos, 1990).

Architecture: To process the simulation sequence only once and answer both mass and force questions, a multibranch neural network as shown in Figure 2 was developed. This Y-shaped architecture is divided in two parts: the bottom layers, shared across the two tasks, and two separate heads, one per task. In the ideal case, the bottom layers will tend to extract features that are useful for both tasks and the top layers will specialise in processing that is task-specific. This does not only lead to a more efficient model that reuses features but also to a more robust one as it combines learnings from two different tasks.

The network is composed of two recurrent layers of 25 units each, followed in each head by another two recurrent layers of the same size. The last hidden state generated by the top recurrent layers is fed into a linear layer which reduces its size to 3 values (one per answer). A softmax function is applied to these values which clamps them between 0 and 1 and are then used to calculate the cross entropy loss for each one of the examples. The network is trained on batches of 128 examples, with a learning rate of $\eta=0.01$ adapted with the Adam optimizer (Kingma \& Ba, 2014) with default parameters $\left(\beta_{1}=0.9, \beta_{2}=0.999\right.$ and $\left.\varepsilon=10^{-8}\right)$. Additionally, to prevent the network from overfitting to the training data, dropout $(\mathrm{p}=0.5)$ is applied to each recurrent layer in the network (Srivastava, Hinton, Krizhevsky, Sutskever, \& Salakhutdinov, 2014) .

\section{Analysing RNNs and their uncertainty}

For a trained RNN, the resulting softmax values (one per answer adding up to 1) are frequently seen as the probability of the example belonging to each class. The entropy of these values can be interpreted as a metric of how confident the network is about its answer. For those examples where the RNN is most undecided, the network will assign similar probabilities to every class, a distribution with a high entropy. Conversely, when it is most certain, one of the classes will accumulate most of the probability, a distribution with low entropy. The maximum entropy value $(\approx 1.58), H_{\text {max }}$, corresponds to a uniform distribution. If we subtract the entropy for a given answer from $H_{\max }$, we obtain the entropy reduction or "certainty", for that example, $\Delta H(\hat{y})$.

Since RNNs can classify sequences of different length, if we feed a short subsequence to the RNN (e.g. 1 second), the entropy of the output will be reduced according to the evidence that can be seen in the interval. Intervals which contain very clear evidence about what the answer is (such as one target puck orbiting around the other because they attract each other) will result in a relatively high certainty. By resetting the RNN's internal state after each interval, we can obtain a metric of how informative each independent interval in the simulation is which we will refer to as RNN's Interval Information.

\section{Ideal Observer model}

Bramley et al. (2018) use an Ideal Observer (IO) approach to infer the latent properties of the simulated worlds. The IO has access to the underlying world dynamics and can simulate forward and see what the world will look like after some time is elapsed. To calculate the likelihood of a world configuration, the IO compares the obtained simulation for this configuration with the observed trajectories for that same period (assuming some perceptual noise). To obtain a gradual measure of similarity, the objects' positions and velocities in the simulation are reset to the observed ones every 10 frames (1/6 s) during the simulation. The similarity of those intervals can then be integrated in time giving the likelihood of the configuration.

The authors then calculate the posterior probability of each configuration and compute the overall posterior uncertainty 

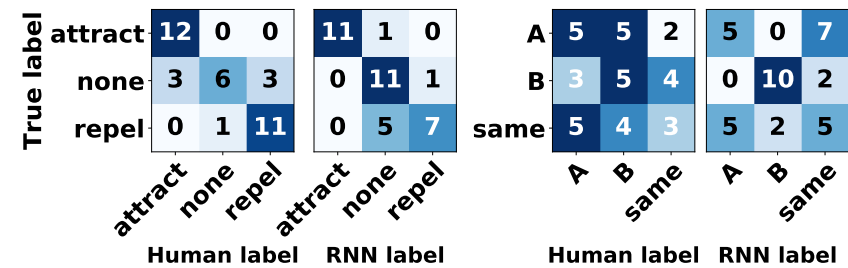

Figure 3: Confusion matrices for the 36 trials in which participants and RNNs were tested. For each trial, the most commonly selected option among participants is used as 'human label'. The same applies for RNN predictions.

for the trial (i.e. the entropy of the posteriors). Then, they subtract the posterior uncertainty from the maximum uncertainty possible (corresponding to an initially uniform distribution over configurations) and obtain the IO posterior confidence for a given simulation. As with the RNN, we will obtain a low posterior uncertainty for those trials where the properties are easy to discern and a higher one for more difficult trials.

Parallel to the final IO confidence, the authors also computed a measure of the evidential value of shorter intervals. In this case, we can compute how informative a given interval is regarding a property by computing its marginal distribution. This measure, called Predictive Divergence (PD), assesses how much the simulations of different configurations of the selected property diverge in the given interval. We will obtain a high PD, for example, for an interval where two target pucks are close as their trajectories will vary greatly depending on whether they attract or repel each other. On the other hand, if the pucks are far apart, the simulation of different configurations will look similar and will result in a low PD value.

\section{Results}

The best performing architecture across all the experiments is the one using GRU cells, only the puck positions and velocities as features, a sequence length of $45 \mathrm{~s}$ sampled at $20 \mathrm{fps}$ giving a total of 900 steps 125 different initialisations of the RNN were trained with this configuration, saving for each one of them the best performing weights for the mass and force questions. These models obtain an average accuracy on the test set of $63.61 \pm 2.33 \%$ and $62.74 \pm 3.2 \%$ for the mass and force questions respectively. These models were then tested on the same trials as participants. For each trial in this test dataset, the percentage of participants and model initialisations guessing it correctly was calculated thus obtaining a total of 36 pairs of percentages for comparison. In Figure 3 . confusion matrices for both human participants and the RNN models can be seen.

Overall, answering force questions is significantly easier than answering mass questions for both participants $(t(36)=$ $4.33, p<0.001)$ and RNN models $(t(36)=1.99, p=0.049)$.

\footnotetext{
${ }^{1}$ See $\overline{\text { Appendix }}$ for a full performance table.
}

For mass questions, the model is significantly better than participants (paired, $t(36)=3.68, p<0.001$ ) achieving $53.66 \% \pm 28.39$ accuracy vs $37.83 \% \pm 14.59$ achieved by the participants. However, as can be appreciated in Figure 3. the difference in performance varies depending on the actual class. More specifically, the RNNs are only significantly better at answering questions belonging to the ' $\mathrm{B}$ ' class (paired, $t(12)=4.85, p<0.001)$. For the force questions, the model is also significantly better (paired $t(36)=2.36, p=0.02$ ) achieving $65.66 \pm 21.32$ against $54.62 \pm 17.68$ for the participants. At class level, similarly as before, this difference is only significant for one class: 'none' (paired, $t(12)=$ $4.78, p<0.001)$.

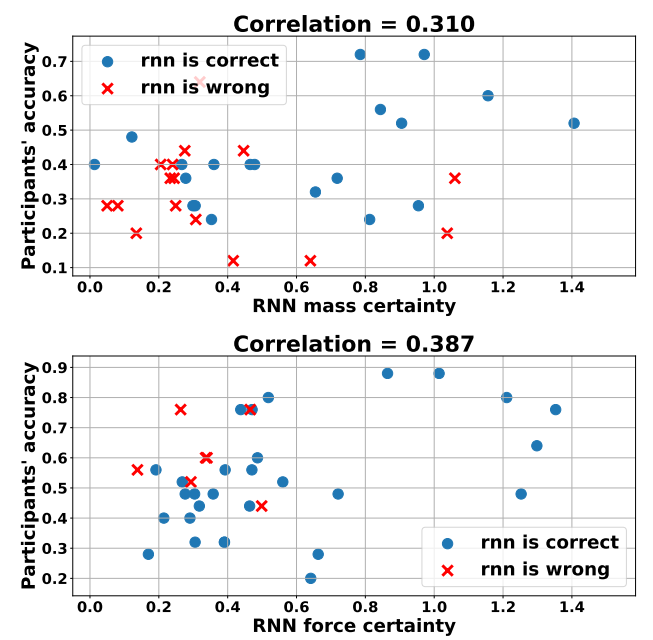

Figure 4: The average RNN certainty and the percentage of participants that correctly label a trial is positively correlated. Each point represents one of the 36 trials.

Across the different trials, the percentage of participants and of RNNs choosing each option is positively correlated for both force questions ( $\rho_{\text {attract }}=0.844, \rho_{\text {none }}=0.446$ and $\left.\rho_{\text {repel }}=0.546\right)$ and mass questions $\left(\rho_{A}=0.548, \rho_{B}=0.590\right.$ and $\rho_{\text {same }}=0.109$ ). Percentages of 'attract' answers are the most strongly correlated while 'same' examples only show a correlation of 0.109 . This correlation can also be found between the softmax output of the RNNs (i.e. the probability that they assigns to each one of the classes) and the percentage of participants choosing that class. The correlation coefficients are similar to those found before: $\rho_{\text {attract }}=0.846$, $\rho_{\text {none }}=0.475$ and $\rho_{\text {repel }}=0.593 ; \rho_{A}=0.571, \rho_{B}=0.599$ and $\rho_{\text {same }}=0.041$.

In Figure 4, the percentage of participants that answer correctly to a trial has been plotted against the RNNs certainty for the same trial. These two values appear to be positively correlated for both question types: 0.387 for force questions and 0.310 for mass questions. In other words, questions where the RNN assigns a large probability value to one of the options often correspond to those where a larger number participants guess correctly. A similar correlation ( 0.382 and 0.396 for force and mass questions respectively) can be found 

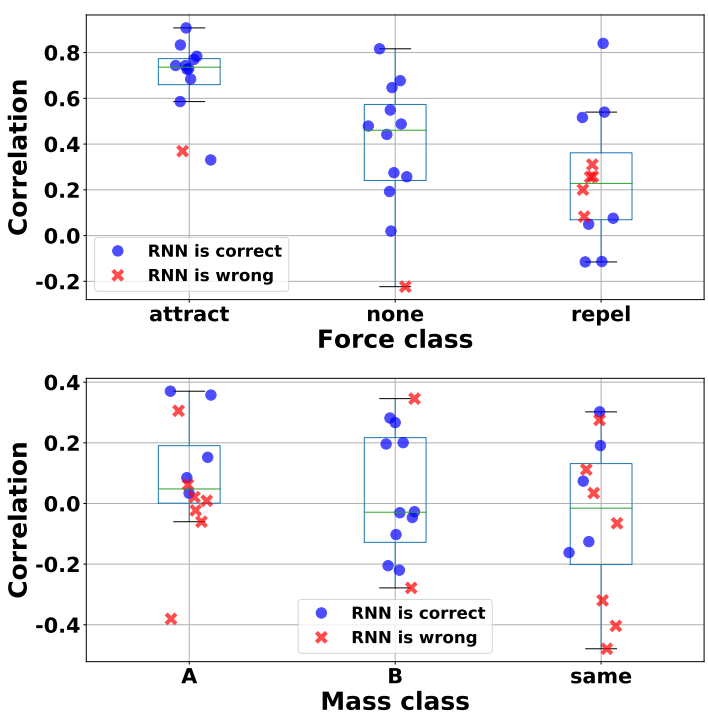

Figure 5: A box plot showing the correlation between PD and RNN's Interval Information. For force evidence (top), they are positively correlated while for mass (bottom) they show varying results.

between the average participant confidence, that is, how sure they are of their answer and the RNN's certainty.

The confidence obtained using the Ideal Observer model is less correlated with participant accuracy for force questions $(\rho=0.187)$ and not correlated at all for mass questions ( $\rho=0.002$ ). If compared with participant confidence, force questions are positively correlated $(\rho=0.318)$ while for mass questions this value is again very close to $0(\rho=-0.064)$. Similarly, between the Ideal Observer's confidence and the RNN certainty there exists a correlation for force $(\rho=0.290)$ but not for mass questions $(\rho=0.044)$.

This difference between the Ideal Observer model and the RNN is also found when comparing the PD with the RNN's information for intervals of $1 \mathrm{~s}$. For force questions, the average correlation between both metrics is $0.436 \pm 0.306$ with a majority of the trials eliciting a positive correlation. On the other hand, for mass trials the average correlation is $0.020 \pm 0.225$. The similarity between these two metrics varies also across classes as can be seen in Figure 5. Force trials of the class 'attract' are the most strongly correlated ones with a majority of values from 0.6 to 0.9 (see Figure 6 for an example), followed by those in the class 'none' (avg. correlation $=0.5$ ) and, finally, those from the class 'repel' (avg. correlation $=0.2$ ). Mass trials do not vary as much across classes: most trials are centered around 0 with some outliers showing positive correlation (with as much as 0.4 ) and others showing negative correlation as far as -0.45 (see Figure 6 for that example).

By taking the Predictive Divergence as ground truth for the evidence (recalling that in using the true simulator the IO model was never systematically wrong but just more or less confident), we can compute for each interval whether the RNN correctly identifies it, misses it or "hallucinates" nonexistent evidence. The RNN correctly captures $56 \pm 12 \%$ of
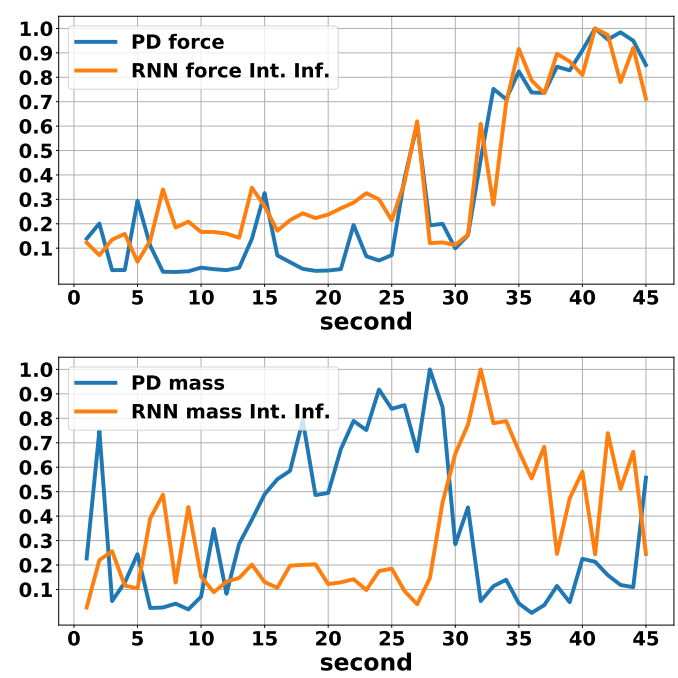

Figure 6: Ideal Observer's Predictive Divergence and RNN's Interval Information compared in two different trials. In the top example, belonging to the class 'attract', they show a positive correlation. In the bottom one, an example of the class 'same', the metrics are negatively correlated.

the force evidence, for $2 \pm 2 \%$ of the intervals it misses the contained evidence and, finally, for $39 \pm 11 \%$ of the intervals it makes up evidence. In comparison, for mass evidence, the RNN correctly captures a significantly higher proportion $63 \pm 11 \%(t(36)=2.45, p=0.016)$ of the evidence and makes up a significantly lower amount of evidence $14 \pm 6 \%$ $(t(36)=11.53, p<0.001)$. However, it also misses significantly more evidence: $19 \pm 10 \%(t(36)=8.67, p<0.001)$.

For additional plots and video replays, see the Appendix.

\section{Discussion}

In this work, we have shown that an RNN model can be trained to achieve as good or better than human performance in a physical reasoning task. This means that, for this task in particular, an explicit simulation model of the environment is not needed to reach human-level inference abilities. Moreover, we have shown that RNNs do not only achieve a closer accuracy to human participants than the model-based approach presented by Bramley et al. (2018) but that they also show prediction patterns similar to those found in participants.

At a high level, both RNNs and participants find questions relating to force easier than mass questions. This contrasts with the IO approach which is significantly more confident about mass questions. As shown, this similarity can be further observed by looking at the percentages of participants and RNN models choosing an answer, which are positively correlated. This means that, for a given trial a comparable number of participants and RNNs will select each possible class ${ }^{2}$ This suggests that both the model-free approach and participants absorb evidence from the clips in a somewhat similar manner which in turn limits their performance alike.

\footnotetext{
${ }^{2}$ Note that this still true for classes with a significant difference in performance ('B' and 'none').
} 
Note that the correlation was particularly low (0.109) for the cases where target objects had the 'same' mass. However, as can be seen in Figure 3, this is primarily due to the poor performance of both models and participants in these examples.

Looking at the probability assigned to each class by the RNNs gives a more fine grained insight into the trials' uncertainty. These probabilities are still strongly correlated with the participant choices. This means that, regardless of the initialisation, RNNs assign a similar probability to each class and that it is very unlikely that two RNN initialisations will assign a very high and very low probability to a class in a given example (since that would lead to a uniform distribution of probabilities). In turn, this suggests that there are easier and more complicated examples for the RNN and, since the probabilities assigned are correlated to the participants' choices, that they match easier and harder examples for the participants.

As shown in Figure 4, these beliefs can be confirmed by checking the relation between the RNNs' certainty with the percentage of participants' guessing an answer correctly. That is, those examples where the RNN is more certain correspond to those with a higher participant accuracy. Also importantly, the reported confidence of the participants on their answer, a more direct metric of their certainty, is also positively correlated with the RNN certainty. All of this suggests that the RNNs and the participants not only have a comparable accuracy on the task and select their options in a similar way but also that they share the certainty about their answers.

In comparison, the IO's confidence does not capture the trial difficulty for participants as precisely. For force trials, the correlation coefficient between the posterior and the participants' accuracy is smaller than the RNNs' ( 0.187 versus 0.387). This difference is even more pronounced for mass examples where the correlation for the IO approach is nonexistent $(0.002$ versus 0.310$)$. The same happens for the participant confidence, with force examples being positively correlated with the IO's confidence $(0.318)$ while the mass ones are not. This evidence shows that assessing trials' difficulty is not trivial and further supports the idea that a model-free approach may be a more accurate representation of how participants reason in the task.

Lastly, we looked at the relationship between the reduction of uncertainty in RNN with the Predictive Divergence and, as can be seen from Figure 6 top, there are examples that closely relate to each other. In general, however, it is not uncommon that the RNN misses or confuses evidence present in the trials. For force questions, the limiting factor for the RNN's performance seems to be that it sometimes misinterprets what it sees as most consistent with a label different from the truth. One reason for this could be the existence of pairwise forces between the target and distractor pucks as, for example, a repulsion exerted by a nearby distractor puck may be seen as a repulsion caused by a target puck. In comparison, for mass questions, the limiting factor seems to be that the RNN is missing evidence. This could be both because of the brevity of evidence from collisions as well as the complexity of gathering evidence from collisions with distractor pucks.

Human participants also confuse or miss evidence and thus it is useful that our chosen model is capable of reproducing this. To further test the similarity between participants, RNNs and the probabilistic model, it would be interesting to modify the environment and see how it affects the performance. Removing distractor forces should not necessarily affect the performance of the probabilistic model while, if our hypothesis is correct, an increment in performance should be seen in both RNN and participants. On the other hand, if we removed the distractor pucks and tested the accuracy on mass questions, the probabilistic model's performance should drop, as a source of information is removed, but the RNN's and the participants' should remain the same.

\section{References}

Allen, K. R., Smith, K. A., \& Tenenbaum, J. B. (2019). The tools challenge: Rapid trial-and-error learning in physical problem solving. arXiv preprint arXiv: 1907.09620.

Baillargeon, R., Li, J., Ng, W., \& Yuan, S. (2009). An account of infants' physical reasoning. Learning and the infant mind, 66-116.

Battaglia, P. W., Hamrick, J. B., \& Tenenbaum, J. B. (2013, November). Simulation as an engine of physical scene understanding. Proceedings of the National Academy of Sciences, 110(45), 18327-18332.

Battaglia, P. W., Pascanu, R., Lai, M., Rezende, D., \& Kavukcuoglu, K. (2016, December). Interaction Networks for Learning about Objects, Relations and Physics. arXiv:1612.00222 [cs]. (arXiv: 1612.00222)

Bramley, N. R., Gerstenberg, T., Tenenbaum, J. B., \& Gureckis, T. M. (2018, September). Intuitive experimentation in the physical world. Cognitive Psychology, 105, 9-38.

Cho, K., van Merrienboer, B., Bahdanau, D., \& Bengio, Y. (2014, September). On the Properties of Neural Machine Translation: Encoder-Decoder Approaches. arXiv:1409.1259 [cs, stat]. (arXiv: 1409.1259)

Davis, E., \& Marcus, G. (2015). The scope and limits of simulation in cognitive models. arXiv preprint arXiv: 1506.04956.

Hamrick, J. B., Battaglia, P. W., Griffiths, T. L., \& Tenenbaum, J. B. (2016). Inferring mass in complex scenes by mental simulation. Cognition, 157, 61-76.

Hespos, S. J., Ferry, A. L., \& Rips, L. J. (2009, May). Five-Month-Old Infants Have Different Expectations for Solids and Liquids. Psychological Science, 20(5), 603-611.

Kingma, D. P., \& Ba, J. (2014, December). Adam: A Method for Stochastic Optimization. arXiv:1412.6980 [cs]. (arXiv: 1412.6980)

Ludwin-Peery, E., Bramley, N. R., Davis, E., \& Gureckis, T. M. (2019, May). Limits on the Use of Simulation in Physical Reasoning (preprint). PsyArXiv.

Ludwin-Peery, E., Bramley, N. R., Davis, E., \& Gureckis, T. M. (in revision) Broken Physics: A Conjunction Fallacy Effect in Intuitive Physical Reasoning.

Marr, D. (1982). Vision. New York: Freeman \& Co.

Mccloskey, M., Caramazza, A., \& Green, B. (1980, December). Curvilinear Motion in the Absence of External Forces: Naïve Beliefs About the Motion of Objects. Science, 210(4474), 1139-1141.

Mottaghi, R., Bagherinezhad, H., Rastegari, M., \& Farhadi, A. (2015, November). Newtonian Image Understanding: Unfolding the Dynamics of Objects in Static Images. arXiv:1511.04048 [cs]. (arXiv: 1511.04048)

Salehinejad, H., Sankar, S., Barfett, J., Colak, E., \& Valaee, S. (2017, December). Recent Advances in Recurrent Neural Networks. arXiv:1801.01078 [cs]. (arXiv: 1801.01078)

Smith, K. A., Dechter, E., Tenenbaum, J. B., \& Vul, E. (2013). Physical predictions over time. In Proceedings of the annual meeting of the cognitive science society (Vol. 35).

Spelke, E. S. (1990). Principles of Object Perception. Cognitive Science, 14(1), 29-56.

Srivastava, N., Hinton, G., Krizhevsky, A., Sutskever, I., \& Salakhutdinov, R. (2014). Dropout: a simple way to prevent neural networks from overfitting. The journal of machine learning research, 15(1), 1929-1958.

Ullman, T. D., Stuhlmüller, A., Goodman, N. D., \& Tenenbaum, J. B. (2018). Learning physical parameters from dynamic scenes. Cognitive Psychology, 104, 57-82.

Van Schaik, C. P., Deaner, R. O., \& Merrill, M. Y. (1999). The conditions for tool use in primates: implications for the evolution of material culture. Journal of Human Evolution, 36(6), 719-741.

Werbos, P. J. (1990). Backpropagation through time: what it does and how to do it. Proceedings of the IEEE, 78(10), 1550-1560. 